\title{
53BP1 expression as a biomarker to differentiate thyroid follicular tumors
}

\author{
Ayako Sato ${ }^{1,2}$, Katsuya Matsuda ${ }^{2}$, Takahiro Motoyama ${ }^{2}$, Zhanna Mussazhanova ${ }^{2,3}$, Ryota Otsubo', \\ Hisayoshi Kondo ${ }^{4}$, Yuko Akazawa ${ }^{2}$, Miyoko Higuchi ${ }^{5}$, Ayana Suzuki ${ }^{5}$, Mitsuyoshi Hirokawa ${ }^{5}$, Akira Miyauchi ${ }^{5}$, \\ Takeshi Nagayasu ${ }^{1}$ and Masahiro Nakashima ${ }^{2}$ \\ 'Department of Surgical Oncology, Nagasaki University Graduate School of Biomedical Sciences, Nagasaki, Japan \\ 2Department of Tumor and Diagnostic Pathology, Atomic Bomb Disease Institute, Nagasaki University Graduate School of Biomedical Sciences, \\ Nagasaki, Japan \\ ${ }^{3}$ Al-Farabi Kazakh National University, Almaty City, Republic of Kazakhstan \\ ${ }^{4}$ Biostatics Section, Division of Scientific Data Registry, Atomic Bomb Disease Institute, Nagasaki University Graduate School of Biomedical Sciences, \\ Nagasaki, Japan \\ ${ }^{5}$ Department of Diagnostic Pathology and Cytology, Kuma Hospital, Kobe, Hyogo, Japan
}

Correspondence should be addressed to M Nakashima: moemoe@nagasaki-u.ac.jp

\begin{abstract}
We have previously reported that the expression of p53-binding protein 1 (53BP1) in nuclear foci (NF), a marker reflecting DNA damage response (DDR), detected using immunofluorescence (IF) is useful to estimate the malignant potency of diverse cancers. In this prospective study, we clarified the impact of 53BP1 expression via IF as a biomarker to differentiate thyroid follicular tumors (FTs) with liquid-based cytology (LBC). A total of 183 consecutively obtained-LBC samples, which were preoperatively suspected as FTs, were analyzed. Before histological diagnosis, the type of 53BP1 immunoreactivity in LBC was classified as follows: low DDR type, one or two NF; high DDR type, three or more NF; large foci type, larger than $1.0 \mu \mathrm{m}$; abnormal type, intense nuclear staining. Among the 183 cases, 136 cases were postoperatively diagnosed as FTs, including adenomatous goiter (AG, $n=30$ ), follicular adenoma (FA, $n=60)$, FT-uncertain malignant potency (FT-UMP, $n=18$ ), and follicular carcinoma (FC, $n=28)$, and 47 cases were diagnosed as tumors other than FTs or technically inadequate materials. Total 136 FT cases were collated with the type of 53BP1 immunoreactivity in LBC. The mean incidence expressing abnormal 53BP1 expression was significantly higher in FC than FA (9.5\% vs 2.6\%, $P$-value $<0.001)$. When adopting $4.3 \%$ as a cut-off value to distinguish FC from FA, the sensitivity, specificity, positive predictive value, and negative predictive value were $89.3,83.3,71.4$, and 94.3\%, respectively. Therefore, IF analysis of 53BP1 expression can be employed as a novel technique to diagnose FTs and to distinguish between different types of FTs using LBC.
\end{abstract} Key Words

- 53BP1

\section{Introduction}

Thyroid follicular carcinoma (FC) must be differentiated from the more common follicular adenoma (FA). However, it is almost impossible to preoperatively diagnose follicular tumors (FTs) by fine-needle aspiration cytology (FNAC) because these lesions can be distinguished based on histological criteria, such as capsular/vascular invasion or metastasis but not on cytologic features, as in the case of papillary carcinoma (PC) $(1,2)$. According to the Bethesda System for Reporting Thyroid Cytology, almost all FC cases are divided into category III (atypia of undetermined significance or follicular lesion of undetermined significance) or category IV (follicular neoplasm or suspicious for a follicular neoplasm) in which the estimated malignancy risk is $5-15 \%$ or $15-30 \%$ (3).

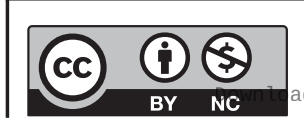


To avoid over-surgery, several patients with high-risk FC, such as angioinvasive, widely invasive, and with poorly differentiated components, require a secondary curative operation after a preceding histological diagnostic thyroidectomy.

The p53-binding protein 1 (53BP1) is a family of evolutionarily conserved DNA damage response (DDR) molecules, which rapidly localize at the sites of DNA double-strand breaks forming nuclear foci (NF) to co-operatively activate p53 together with other DDR molecules, such as $\gamma \mathrm{H} 2 \mathrm{AX}$ and ATM $(4,5,6)$. Previously, we demonstrated using immunofluorescence (IF) that the profile of 53BP1 expression, including the number of NF, which reflects the DDR, is clearly altered during tumorigenesis of a diverse cancers, such as thyroid, skin, cervix, bladder, and esophageal carcinomas $(7,8,9,10$, 11). Our more recent study using IF analysis of formalinfixed paraffin-embedded (FFPE) tissues demonstrated that the number of 53BP1-positive NF increases along with higher malignant potential of thyroid FTs, such as FA, minimally invasive FC, and widely invasive FC (12). Thus, IF-determined 53BP1 expression profile in FTs can be an attractive candidate biomarker to distinguish FC from FA in FFPE tissues. Indeed, when we adopted 3.1\% as a cut-off value for the incidence of high DDR type, this test could differentiate FC from FA among 69 FFPE FT samples with a sensitivity of $90.5 \%$ and a specificity of $77.8 \%$ (12).

Tumor tissue consists of heterogeneous cells, in terms of both cell cycle and sensitivity to DDR. Human cells, from normal to cancer cells, are continuously exposed to numerous genotoxic agents, both exogenously and endogenously, and consequently show variable 53BP1 expression profiles, reflecting the status of the DDR machinery and/or sensitivity to DDR, which could be associated with the level of malignant potential. This working hypothesis is illustrated in Fig. 1. Therefore, the present study aimed to prospectively clarify the impact of 53BP1 expression, which was analyzed by IF, as a biomarker to differentiate FTs using liquid-based cytology (LBC). Our findings indicate that IF analysis of 53BP1 expression will not only be an auxiliary histologic technique to accurately diagnose FTs but also a novel technique to enable diagnosis using LBC from FTs.

\section{Materials and methods}

\section{Subjects}

A total of 187 consecutive cases were clinically and cytologically suspected as FTs (category III or IV of the Bethesda System) before surgery at Kuma Hospital in Kobe between August 2018 and July 2019. Of these, 183 cases were available in this study. The remaining four cases were excluded from the study because the subjects declined to join in this study. All cytology samples were obtained from surgically resected thyroid glands by FNAC, and sent to the Department of Tumor and Diagnostic Pathology, Nagasaki University. Final diagnosis of all cases was histologically confirmed at Kuma Hospital. LBC specimens were prepared for conventional Papanicolaou staining to confirm the presence of FT cells from FNA samples, and used to quantitatively analyze 53BP1 expression in FT cells by IF at Nagasaki University. Exclusion criteria were, (i) technically inadequate samples, such as either too many cells showing severe nuclear overlapping (unable to identify the number of NF) or too few cells (less than 10 clusters of follicular cells) to evaluate type of 53BP1 expression, (ii) cases finally diagnosed as

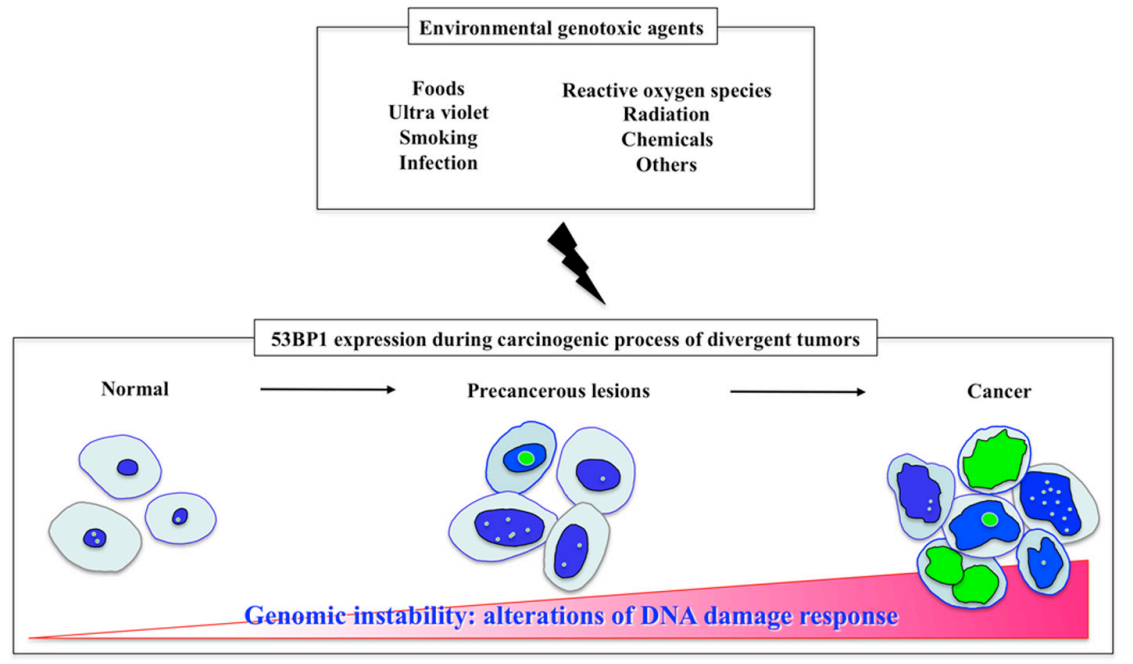

\section{Figure 1}

A schematic representation of our working hypothesis. Tumor tissues consist of heterogeneous cells, in terms of sensitivity to DNA damage response (DDR). Human cells are continuously exposed to environmental genotoxic agents, exogenously and endogenously, and consequently show a variable pattern of 53BP1 expression, reflecting the status of DDR machinery or sensitivity of DDR, which is associated with the level of malignant potential. Blue and green colors show nuclei and staining of p53-binding protein 1 (53BP1) via immunofluorescence, respectively. https://ec.bioscientifica.com https://doi.org/10.1530/EC-20-0630 (c) 2021 The authors Published by Bioscientifica Ltd

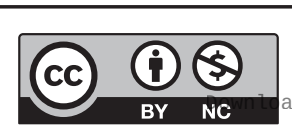

This work is licensed under a Creative Commons Attribution-NonCommercial 4.0 International License. ded from Bioscientifica.com at 04/26/2023 12:03:12PM via free access 
papillary carcinoma, well differentiated tumor-uncertain malignant potency (UMP), poorly differentiated carcinoma, and other nodular lesions by histopathology. The 53BP1 expression profile in each case was determined before obtaining their clinicopathological information from Kuma Hospital. A schematic representation of these analytic procedures is shown in Fig. 2.

This study was conducted in accordance with the tenets of the Declaration of Helsinki and approved by the institutional ethical committee for medical research at both Nagasaki University (approval date: June 20, 2019; \#15062617-3) and Kuma Hospital (approval date: July 6, 2017; \#170706-4). Profiles of all patients were anonymized by coding, and collectively summarized with the obtained data as final datasets.

\section{Analysis of 53BP1 expression profile by IF}

FNA samples were stored in preservative solution (CytoRich $^{\mathrm{TM}}$ Red Preservative, Japan Becton Dickinson Corp., Tokyo, Japan) for BD SurePath ${ }^{\mathrm{TM}}-\mathrm{LBC}$ preparation (Japan Becton Dickinson Corp.). Preserved cells were manually prepared for LBC slides according to the manufacturer's instruction. After washing with $0.01 \mathrm{M}$ PBS (pH 7.4), LBC slides were preincubated with $10 \%$ $(v / v)$ normal goat serum. Samples were then incubated with anti-53BP1 rabbit polyclonal antibody (Bethyl Labs, Montgomery, TX, USA) at 1:1000 dilution for $1 \mathrm{~h}$ at $24^{\circ} \mathrm{C}$. The slides were subsequently incubated with Alexa Fluor 488-conjugated goat anti-rabbit antibody (Molecular Probes Inc.). Specimens were counter-stained with DAPI dihydrochloride (Vysis Inc., Downers Grove, IL, USA), analyzed, and photographed using a High Standard All-in-One Fluorescence Microscope (Biorevo BZ-X710; KEYENCE Japan, Osaka, Japan) with the Z-stack mode, which accumulated images from 20 to 30 slices. IF analyses focused on parts of sheet-like arranged cells in both small and large clusters, which are considered as being originated from follicular epithelium. Signals were analyzed in more than ten viewing areas per case at a 1000-fold magnification. All signals for 53BP1 expression were measured using the image analysis software (BZ-X analyzer) provided with the Biorevo BZ-X710 microscope. Expression of 53BP1 was classified into three types as shown in Fig. 3: (i) low DDR type: none, one or two discrete NF, (ii) high DDR type: three or more discrete NF, or discrete NF that are larger than $1.0 \mu \mathrm{m}$ in the minor axis, (iii) abnormal type: intense and heterogenous expression. The percentage of follicular cells displaying each pattern of 53BP1 expression was calculated in each case.

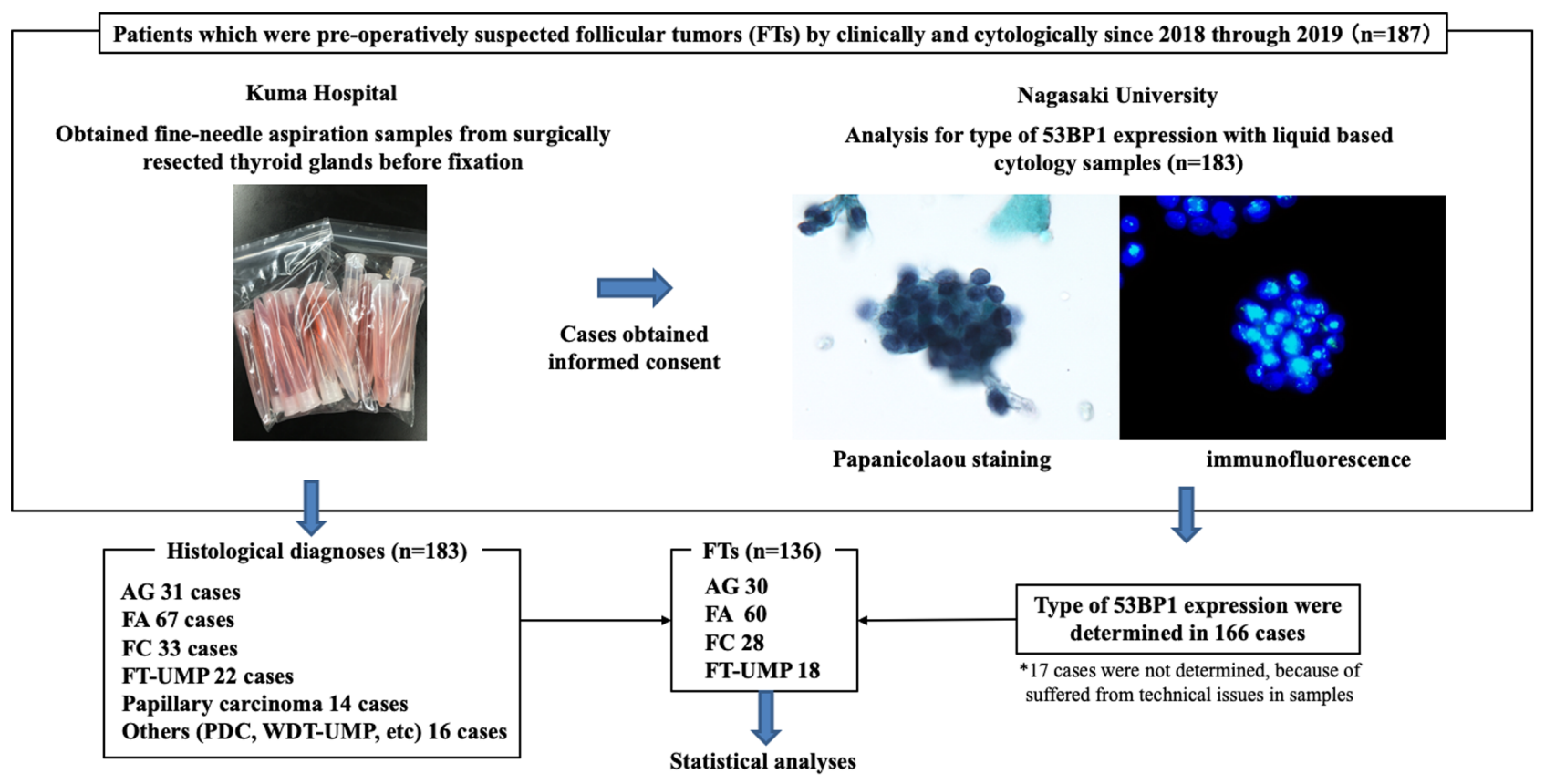

Figure 2

Schematic representation of analytic procedures in this study. 53BP1, p53-binding protein 1; LBC, liquid-based cytology; AG, adenomatous goiter; FA, follicular adenoma; FC, follicular carcinoma; FT-UMP, follicular tumor-uncertain malignant potential; PDC, poorly differentiated carcinoma; WDT-UMP, well differentiated tumor-UMP.

https://ec.bioscientifica.com

https://doi.org/10.1530/EC-20-0630 (c) 2021 The authors Published by Bioscientifica Ltd

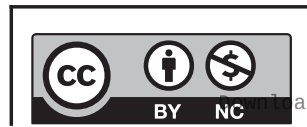

This work is licensed under a Creative Commons Attribution-NonCommercial 4.0 International License. ded from Bioscientifica.com at 04/26/2023 12:03:12PM 


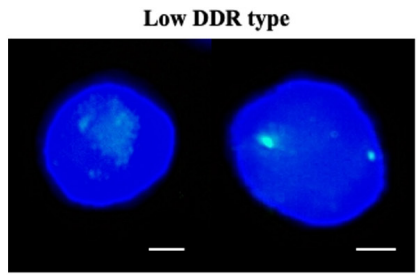

$\mathrm{NF} \leq 2$

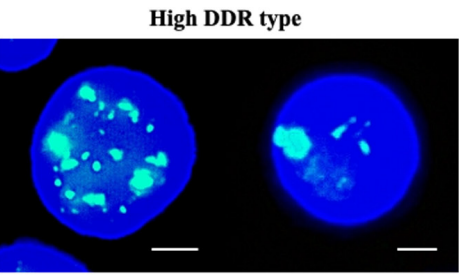

$\mathbf{N F} \geq 3$

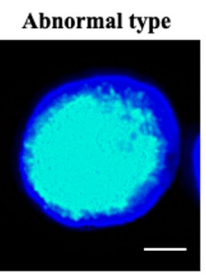

Intense heterogeneous

\section{Figure 3}

Pattern of p53-binding protein 1 (53BP1)

expression in liquid-based cytology samples determined via immunofluorescence. Low DNA damage response (DDR) type, faint nuclear staining or one or two discrete nuclear foci (NF); high DDR type, three or more discrete NF or NF larger than $1.0 \mu \mathrm{m}$; abnormal type, intense, and heterogeneous nuclear staining. The scale bars indicate $2 \mu \mathrm{m}$.

\section{Statistical analysis}

To assess association between the histological type of FTs and gender, the Cochran-Armitage test was used. The Jonckheere-Terpstra test was used to assess associations between the histological type of FTs and differences in the pattern of 53BP1 expression. This statistical test is a rankbased nonparametric test that can be used to determine if there is a statistically significant trend between an ordinal independent variable (the pattern of 53BP1 expression) and a continuous or ordinal dependent variable (the histological type of FTs, such as $\mathrm{AG}<\mathrm{FA}<\mathrm{FT}-\mathrm{UMP}<\mathrm{FC}$ ). Differences in the frequency of the abnormal type of 53BP1 expression between FA and FC were evaluated using Student's $t$-test. Differences in the frequency of each type of 53BP1 expression between gender were also evaluated using Student's $t$-test. The significance of 53BP1 expression by IF as a diagnostic test was evaluated by logistic regression model and receiver operating characteristic (ROC) curve. All statistical analyses were performed using SAS software (version 9.4, SAS Institute Inc., Cary, NC, USA) with statistical significance set at values less than 0.05 .

\section{Results}

\section{Clinicopathological profiles of the evaluated cases}

IF analysis of 53BP1 immunoreactivity was carried out on all 183 cases. Seventeen cases were excluded from the study due to exclusion criteria (i), and 30 cases were also excluded due to exclusion criteria (ii). The other 136 cases were histologically diagnosed as FTs, including AG (30 cases: $22.1 \%), 60$ cases (44.1\%) of FA, 18 (13.2\%) of FT-UMP, and 28 (20.6\%) of FC, and were collated with the pattern of 53BP1 expression in LBC by IF.

\section{Abnormal types of 53BP1 expression as an indicator to detect FC}

Experimental results from the evaluated cases, including 53BP1 expression profiles, are summarized in Table 1 . We found a significant difference in the type of 53BP1 expression among the different histological types of FTs. The mean frequencies of follicular cells expressing abnormal type were 1.8, 2.6, 5.0, and 9.5\% in AGs, FAs, FT-UMPs, and FCs, respectively. Representative images of the 53BP1 staining pattern obtained through IF are shown in Fig. 4 and Supplementary Fig. 1 (see section on supplementary materials give at the end of this article). The Jonckheere-Terpstra test revealed significant association between 53BP1 expression profile and histological type of FTs $(P<0.0001)$ (Table 1$)$. The frequency of low DDR type significantly decreased, while that of abnormal type significantly increased in the following order: AGs, FAs, FT-UMPs, minimally invasive (mi) FCs, and widely invasive (wi) or angioinvasive (ai) FCs. The CochranArmitage test revealed no significant differences between

Table 1 The mean frequency of each type of 53BP1 expression in liquid-based cytology samples from follicular tumors detected by immunofluorescence.

\begin{tabular}{|c|c|c|c|c|c|c|c|c|}
\hline & \multirow[b]{2}{*}{$n$} & \multirow[b]{2}{*}{ Mean age (range) } & \multirow[b]{2}{*}{$\mathbf{M} / \mathbf{F}$} & \multirow{2}{*}{$\begin{array}{c}\text { Total } \\
\text { nuclei }\end{array}$} & \multirow[b]{2}{*}{ Nuclei/case (range) } & \multicolumn{3}{|c|}{ Type of 53BP1 expression } \\
\hline & & & & & & Low DDR (\%) & High DDR (\%) & Abnormal (\%) \\
\hline AG & 30 & $60.7(29-85)$ & $5 / 25$ & 14,581 & $486(93-1028)$ & 82.4 & 15.7 & 1.8 \\
\hline FA & 60 & $54.0(20-82)$ & $12 / 48$ & 47,295 & 788 (50-1060) & 79.7 & 17.8 & 2.6 \\
\hline FT-UMP & 18 & $50.6(28-78)$ & $7 / 11$ & 8029 & 446 (113-756) & 72.1 & 13.9 & 5.0 \\
\hline $\mathrm{FC}$ & 28 & $55.3(14-85)$ & $7 / 21$ & 13,774 & $492(80-1015)$ & 70.6 & 19.8 & 9.5 \\
\hline $\mathrm{mi}$ & 9 & $53.9(19-77)$ & $1 / 8$ & 4019 & $467(150-1015)$ & 76.6 & 14.3 & 9.1 \\
\hline \multirow[t]{2}{*}{ wi and ai } & 19 & $53.8(14-83)$ & $5 / 14$ & 9755 & $513(80-975)$ & 68.2 & 22.2 & 9.6 \\
\hline & 136 & 55.0 & $31 / 105$ & 83,679 & 514 & \multicolumn{3}{|c|}{$P<0.0001$ by Jonckheere-Terpstra test } \\
\hline
\end{tabular}

AG, adenomatous goiter; ai, angioinvasive; DDR, DNA damage response; F, female; FA, follicular adenoma; FC, follicular carcinoma; FT-UMP, follicular tumor-uncertain malignant potential; $\mathrm{M}$, male; mi, minimally invasive; wi, widely invasive . 

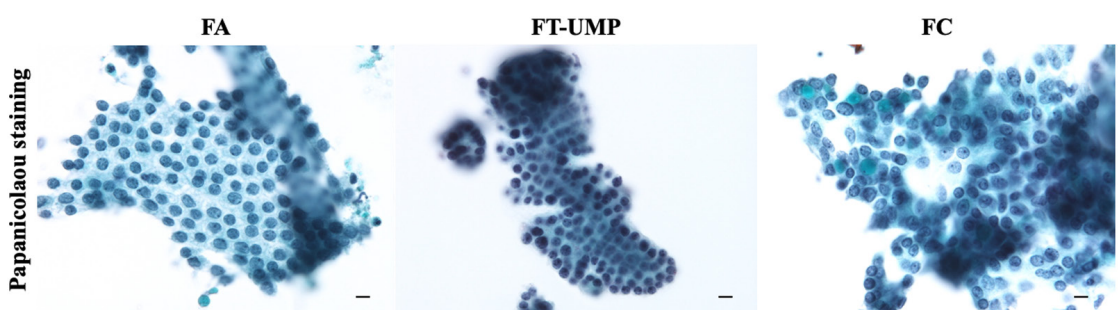

\section{Figure 4}

Cytological features revealed by Papanicolaou staining (upper) and p53-binding protein 1 (53BP1) expression evaluated using immunofluorescence (lower) in liquid-based cytology samples from thyroid follicular tumors. Representative images are provided from follicular adenoma (FA), follicular tumor-uncertain malignant potential (FT-UMP), and follicular carcinoma $(F C)$, respectively. The scale bars indicate $10 \mu \mathrm{m}$.

gender and type of FTs $(P=0.515)$. Also, Student's $t$-test demonstrated no significant differences between gender and type of 53BP1 expression (in cases with low DDR type, $P=0.534$; with high DDR type, $P=0.643$; with abnormal type, $P=0.500$ ). Subsequently, we focused on the ratio of nuclei with abnormal type as an indicator to distinguish the FCs from FAs in LBC samples, and examined the ROC curve for the ratio of nuclei with abnormal type (Fig. 5). The area under the curve (AUC) value was 0.901 (95\% CI, 0.832 to $0.971, P<0.001$ assessed by Mann-Whitney $U$-test),

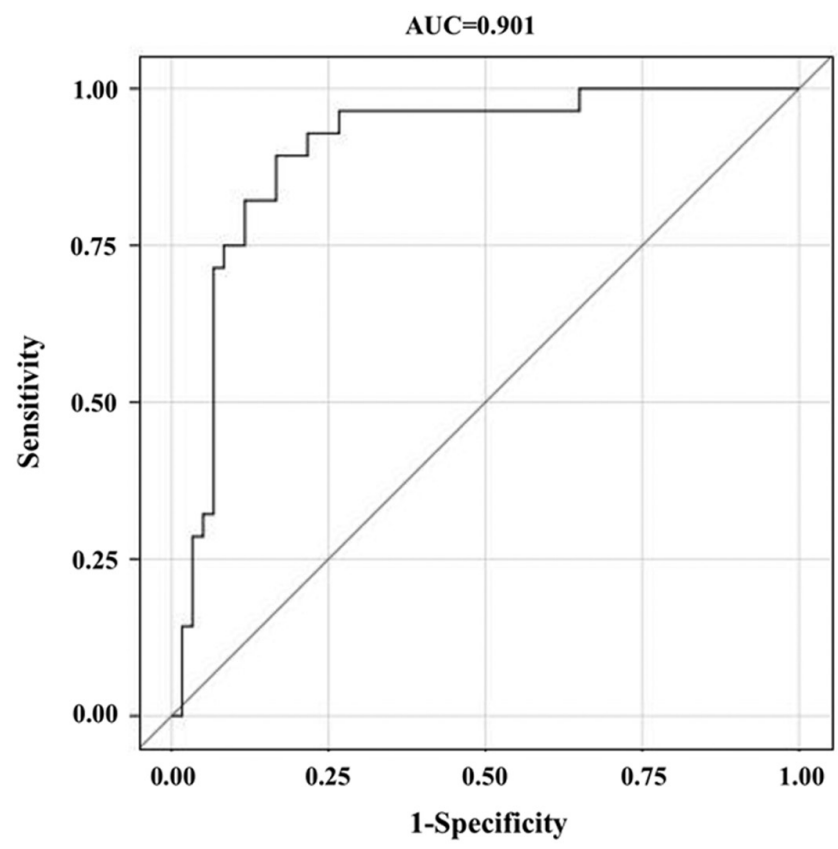

\section{Figure 5}

Receiver operating characteristic (ROC) curve for detecting follicular carcinomas (FCS). The area under the curve (AUC) was $0.901(95 \% \mathrm{Cl}$, 0.832 to $0.971, P<0.001$ assessed by Mann-Whitney $U$-test). The cut-off value of the ratio of nuclei with abnormal type of 53BP1 expression to distinguish FC from follicular adenoma was $4.3 \%$. The sensitivity and specificity were 89.3 and $83.3 \%$, respectively. suggesting the reliable detection of FCs. When adopting a cut-off value of $4.3 \%$ for abnormal type of 53BP1 expression to diagnose FC, the sensitivity, specificity, positive predictive value, and negative predictive value were $89.3,83.3,71.4$, and $94.3 \%$, respectively (Table 2 ).

\section{Discussion}

In this study, using IF, we clearly demonstrated the existence of different patterns of 53BP1 expression in LBC samples from resected FTs, particularly highlighting differences in the frequency of tumor cells with abnormal expression between FA and FC. The mean ratio of abnormal type of 53BP1 immunoreactivity in follicular cells seemed to increase with FT progression, as illustrated in the FA - FT-UMP - FC sequence.

Double IF analysis also revealed the frequent co-localization of 53BP1 and $\gamma \mathrm{H} 2 \mathrm{AX} \mathrm{NF}$ in irradiated rat thyroid glands, suggesting specific detection of 53BP1 $\mathrm{NF}$ at the sites of DNA double-strand breaks induced by irradiation on FFPE sections (Supplementary Fig. 2). With the hypothesis that the presence of 53BP1 NF can be an indicator of endogenous DNA double-strand breaks and DDR activation in individual cells, we previously demonstrated that the type of 53BP1 expression profiles

Table 2 Test performance of immunofluorescence analysis for abnormal types of 53BP1 expression in liquid-based cytology samples to distinguish follicular carcinoma (FC) from follicular adenoma (FA).

\begin{tabular}{|c|c|c|}
\hline Abnormal type & $\mathbf{F A}(n=60 \%)$ & FC $(n=28 \%)$ \\
\hline$\geq 4.3 \%$ & $10(16.7)$ & 25 (89.3) \\
\hline$<4.3$ & $50(83.3)$ & $3(10.7)$ \\
\hline
\end{tabular}

AUC 0.901 (95\% Cl: 0.832-0.971), $P<0.001$ (Mann-Whitney U-test).

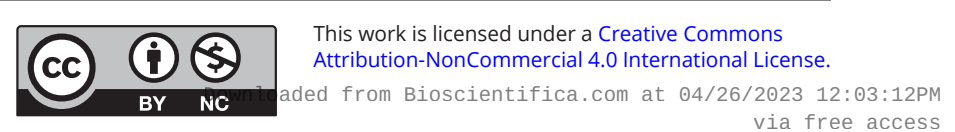


determined by IF may be useful to estimate GIN during a divergent carcinogenesis in thyroid, skin, uterine cervix, bladder, and esophageal cancer $(7,8,9,10,11)$. Classification of 53BP1 expression into the following five categories: (i) stable (no or faint staining); (ii) low DDR (one or two discrete NF); (iii) high DDR (three or more discrete NF); (iv) large focus (discrete NF $\geq 1 \mu \mathrm{m}$ and (v) diffuse (intense and heterogenous expression), showed that both the high DDR and large focus types are closely associated with a higher level of cervical carcinogenesis and high-risk human papilloma virus infection $(9,13)$. Instead, both large focus and diffuse types are significantly associated with high-grade urothelial carcinoma showing chromosomal instability and poor prognosis (10). Moreover, in a rare case of uterine dedifferentiated leiomyosarcoma arising from leiomyoma, we observed that the diffuse 53BP1 expression was restricted only to the sarcoma component, and frequently co-localized with p53 overexpression in sarcoma cells, including highly atypical multinucleated giant cells, suggesting aneuploidy (14). Thus, our analyses revealed that although there is variation in 53BP1 expression among divergent tumors, the high DDR, large focus, and diffuse types of 53BP1 expression were closely associated with high-grade malignancy.

According to the 2017 Bethesda System for Reporting Thyroid Cytology, for category III or IV cases, molecular testing is usually recommended to obtain further diagnostic information. Next-generation sequencing-based ThyroSeq ${ }^{\circledR}$ using FNAC samples was recently reported as the most accurate test for thyroid nodules, which prevents the largest number of diagnostic thyroidectomy cases, avoiding surgery for up to $61 \%$ of all Bethesda III/IV nodules and $82 \%$ of indeterminate nodules with benign pathology (15). The ThyroSeq v3 panel detects known thyroid cancer-associated molecular alterations in 112 genes. The authors suggested that ThyroSeq v3 was able to classify cancers correctly, showing a sensitivity of $94 \%$, a specificity of $82 \%$, a positive predictive value of $66 \%$, and a negative predictive value of $97 \%$ (15).

Our prospective study suggests that the frequency of abnormal 53BP1 expression in FTs can be an attractive candidate biomarker to distinguish FC from FA. Using a cut-off value of $4.3 \%$ for the incidence of abnormal type, this test could differentiate FC or FA among 88 LBC samples from FTs with a sensitivity of $89.3 \%$, a specificity of $83.3 \%$, a positive predictive value of $71.4 \%$, and a negative predictive value of $94.3 \%$. Interestingly, the mean incidence of abnormal type in FT-UMP was found to be the intermediate ratio between the incidences of FA and FC, suggesting its intermediate malignant potency as a follicular neoplasia. Furthermore, the incidences of high DDR and abnormal types were higher in widely invasive/ angioinvasive FCs than in the minimally invasive FC. Therefore, our study suggests that IF analysis of 53BP1 expression is a novel diagnostic method to estimate the malignant potential of FTs with LBCs. We hypothesize that the pattern of 53BP1 expression can be a hallmark of GIN in tumor cells. IF analysis of 53BP1 expression, which requires a much cheaper expense compared with other techniques such as next-generation sequencing and gene expression classification, will not only be an auxiliary histologic technique to accurately diagnose FTs but also a novel technique to make diagnoses using FNA. Moreover, because it is technically possible to automate the quantification of DDR by using single cell imaging, our method may become available to intra-operative diagnosis for FTs. A limitation of this study is that the LBC samples are obtained from surgically resected tumors, many of which were suspected to be FTs based on clinical and cytological evidence preoperatively, but not from preoperative cytology. Further studies using pre-operative FNAC samples are therefore required to elucidate this challenging idea in the near future.

\section{Supplementary materials}

This is linked to the online version of the paper at https://doi.org/10.1530/ EC-20-0630.

\section{Declaration of interest}

The authors declare that there is no conflict of interest that could be perceived as prejudicing the impartiality of the research reported.

\section{Funding}

This work was supported in part through the Atomic Bomb Disease Institute, Nagasaki University; the Grant-in-Aid for Scientific Research from the Japanese Ministry of Education, Science, Sports and Culture (grant numbers 24590414, 26461951); and the Program of the Network-Type Joint Usage/Research Center for Radiation Disaster Medical Science.

\section{Author contribution statement}

M N conceptualized the study; A Sato, K M, T M and Z M performed the experiments; $\mathrm{M} \mathrm{H}, \mathrm{M} \mathrm{H}$ and A Suzuki confirmed the histological and cytological diagnosis; A Sato, and K M analyzed the data; A Sato, K M and M $\mathrm{N}$ drafted the manuscript; $\mathrm{H}$ K performed the statistical analyses; $\mathrm{R} O$., A Y., $\mathrm{A} \mathrm{M}$ and $\mathrm{T} \mathrm{N}$ assisted in the interpretation of the data. All authors read and approved the final version of the manuscript prior to submission.

\section{Acknowledgement}

We would like to thank Editage (www.editage.com) for their writing support on this manuscript.
This work is licensed under a Creative Commons Attribution-NonCommercial 4.0 International License. ded from Bioscientifica.com at 04/26/2023 12:03:12PM 


\section{References}

1 Suster S. Thyroid tumors with a follicular growth pattern: problems in differential diagnosis. Archives of Pathology and Laboratory Medicine 2006130 984-988. (https://doi.org/10.1043/15432165(2006)130[984:TTWAFG]2.0.CO;2)

2 McHenry CR \& Phitayakorn R. Follicular adenoma and carcinoma of the thyroid gland. Oncologist 201116 585-593. (https://doi. org/10.1634/theoncologist.2010-0405)

3 Cibas ES \& Ali SZ. The Bethesda system for reporting thyroid cytopathology. Thyroid 200919 1159-1165. (https://doi.org/10.1089/ thy.2009.0274)

4 Schultz LB, Chehab NH, Malikzay A \& Halazonetis TD. p53 binding protein 1 (53BP1) is an early participant in the cellular response to DNA double-strand breaks. Journal of Cell Biology 2000151 1381-1390. (https://doi.org/10.1083/jcb.151.7.1381)

5 Wang B, Matsuoka S, Carpenter PB \& Elledge SJ. 53BP1, a mediator of the DNA damage checkpoint. Science 2002298 1435-1438. (https:// doi.org/10.1126/science.1076182)

6 Negrini S, Gorgoulis VG \& Halazonetis TD. Genomic instability-an evolving hallmark of cancer. Nature Reviews: Molecular Cell Biology 201011 220-228. (https://doi.org/10.1038/nrm2858)

7 Nakashima M, Suzuki K, Meirmanov S, Naruke Y, MatsuuMatsuyama M, Shichijo K, Saenko V, Kondo H, Hayashi T, Ito M, et al. Foci formation of p53-binding protein 1 in thyroid tumors: activation of genomic instability during thyroid carcinogenesis. International Journal of Cancer 2008122 1082-1088. (https://doi. org/10.1002/ijc.23223)

8 Naruke Y, Nakashima M, Suzuki K, Matsuu-Matsuyama M, Shichijo K, Kondo H \& Sekine I. Alteration of p53-binding protein 1 expression during skin carcinogenesis: association with genomic instability. Cancer Science 200899 946-951. (https://doi.org/10.1111/ j.1349-7006.2008.00786.x)

9 Matsuda K, Miura S, Kurashige T, Suzuki K, Kondo H, Ihara M, Nakajima H, Masuzaki H \& Nakashima M. Significance of p53-binding protein 1 nuclear foci in uterine cervical lesions: endogenous DNA double strand breaks and genomic instability during carcinogenesis. Histopathology 201159 441-451. (https://doi. org/10.1111/j.1365-2559.2011.03963.x)

10 Matsuda K, Kawasaki T, Akazawa Y, Hasegawa Y, Kondo H, Suzuki K, Iseki M \& Nakashima M. Expression pattern of p53-binding protein 1 as a new molecular indicator of genomic instability in bladder urothelial carcinoma. Scientific Reports 20188 15477. (https://doi. org/10.1038/s41598-018-33761-9)

11 Ueki N, Akazawa Y, Miura S, Matsuda K, Kurohama H, Imaizumi T, Kondo H \& Nakashima M. Significant association between 53 BP1 expression and grade of intraepithelial neoplasia of esophagus: alteration during esophageal carcinogenesis. Pathology, Research and Practice 2019215 152601. (https://doi.org/10.1016/j. prp.2019.152601)

12 Otsubo R, Matsuda K, Mussazhanova Z, Sato A, Matsumoto M, Yano H, Oikawa M, Kondo H, Ito M, Miyauchi A, et al. A novel diagnostic method for thyroid follicular tumors based on immunofluorescence analysis of p53-binding protein 1 expression: detection of genomic instability. Thyroid 201929 657-665. (https:// doi.org/10.1089/thy.2018.0548)

13 Kawashita S, Matsuda K, Kondo H, Kitajima Y, Hasegawa Y, Shimada T, Kitajima M, Miura K, Nakashima M \& Masuzaki H. Significance of p53-binding protein 1 nuclear foci in cervical squamous intraepithelial lesions: association with high-risk human papillomavirus infection and p16INK4a expression. Cancer Control 202027 1073274819901170. (https://doi. org/10.1177/1073274819901170)

14 Matsuda K, Akazawa Y, Yamaguchi Y, Mussazhanova Z, Kurohama H, Ueki N, Kohno M, Fukushima A, Kajimura I, Hiraki H, et al. Immunofluorescence analysis of DNA damage response protein p53-binding protein 1 in a case of uterine dedifferentiated leiomyosarcoma arising from leiomyoma. Pathology, Research and Practice 2019215 152640. (https://doi.org/10.1016/j. prp.2019.152640)

15 Steward DL, Carty SE, Sippel RS, Yang SP, Sosa JA, Sipos JA, Figge JJ, Mandel S, Haugen BR, Burman KD, et al. Performance of a multigene genomic classifier in thyroid nodules with indeterminate cytology: a prospective blinded multicenter study. JAMA Oncology 20195 204-212. (https://doi.org/10.1001/jamaoncol.2018.4616)

Received in final form 22 January 2021

Accepted 10 February 2021

Accepted Manuscript published online 19 February 2021 (c) 2021 The authors Published by Bioscientifica Ltd
This work is licensed under a Creative Commons Attribution-NonCommercial 4.0 International License. ded from Bioscientifica.com at 04/26/2023 12:03:12PM 